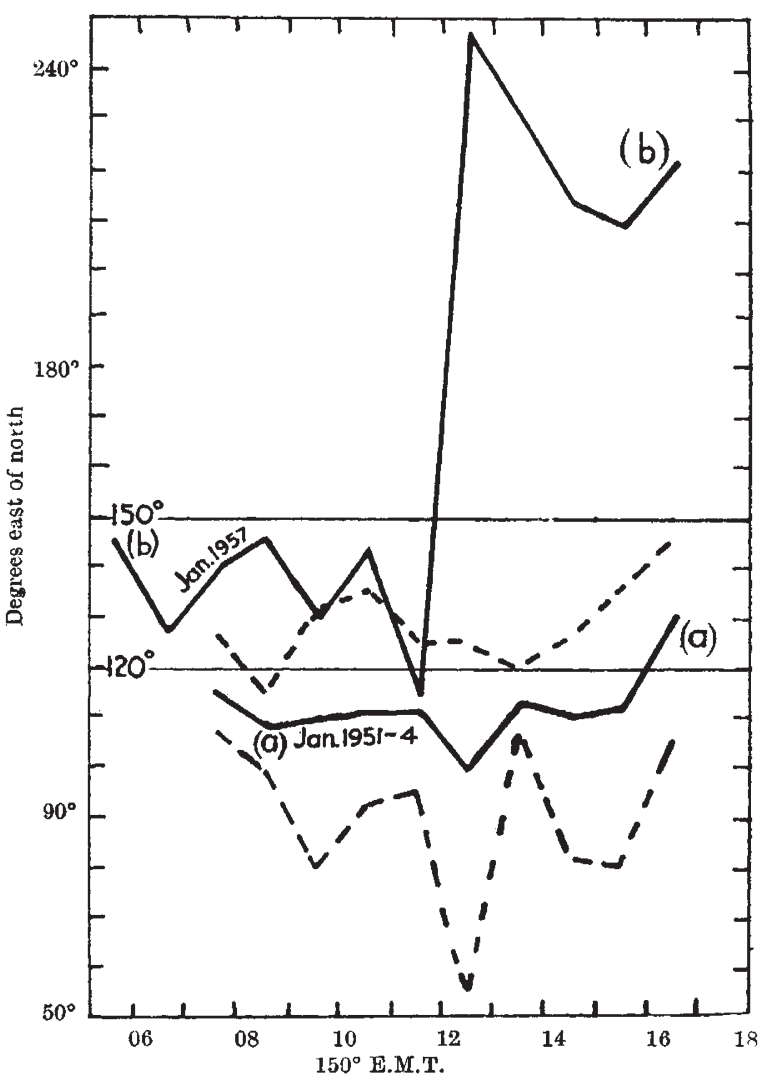

Fig. 1. Diurnal variations in direction of travelling ionospheric disturbances : (a) January 1951-54 (pecked curves show extreme values); (b) January 1957

activity in 1954, and which had already reached a record high value in the (southern) summer of 1956-57.

The assistance of the staff of the Sydney Section in collecting and processing these observations is gratefully acknowledged.

Commonwealth Scientific and

Industrial Research Organization,

Radio Research Laboratories, Sydney Section,

Electrical Engineering Dept., University of Sydney.

' Munro, G. H., Nature, 171, 693 (1953).

\section{Radio Noise from Planets}

THE reception of high-frequency radio noise from the planets Jupiter and Venus ${ }^{1-3}$ has led to speculation on the nature of the causative disturbances. One suggestion is that the noise originates in electrical discharges analogous to terrestrial lightning, and $\mathrm{Kraus}^{3}$, from consideration of the total energy in lightning discharges, has concluded that a source on Venus, of comparable size to those on Earth, would be sufficient to explain the noise. Before the analogy is pressed too far, however, it is desirable to compare the characteristics of the noise in more detail.

One feature of terrestrial lightning is that while the greater part of the energy, and in particular the electromagnetic radiation at very low frequencies, emanates from the high-current stroke to ground, noise at high frequencies has its origin mainly in discharges in the cloud. These cloud discharges are often associated with ground strokes, but are of different form and much lower energy. Any deriva. tion of the high-frequency radiation from consideration of the total energy of the lightning flash may, therefore, be grossly in error. A more satisfactory basis of comparison is the observed form of high-frequency radiation from terrestrial lightning.

Typical noise from a single flash, as observed in a bandwidth of $500 \mathrm{c} / \mathrm{s}$. at $10 \mathrm{Mc}$./s., consists of a burst lasting up to one second and of peak amplitude about $\mathrm{l} \mathrm{mV} . / \mathrm{m}$. at a distance of five kilometres from the flash. These are approximate figures, but only orders of magnitude are being considered. On this basis a similar flash on Venus would give a burst, received on Earth with a power flux of $10^{-26}$ watts $/ \mathrm{m} .{ }^{2} / \mathrm{c}$. $/ \mathrm{s}$., and the corresponding figure for Jupiter would be $5 \times 10^{-28}$ watts $/ \mathrm{m} .{ }^{2} / \mathrm{c} . / \mathrm{s}$.

The measured amplitudes of the noise bursts from Venus and Jupiter are $9 \times 10^{-22}$ and $2.6 \times 10^{-21}$ respectively, at $27 \mathrm{Mc} / \mathrm{s}$. (ref. 3 and private communication from J. D. Kraus). Since a terrestrial atmospheric would have lower power at $27 \mathrm{Mc} . / \mathrm{s}$. than at $10 \mathrm{Mc} / \mathrm{s}$., perhaps by a factor of ten, the discrepancy between the measured noise power and that which would be expected from a flash of terrestrial form is of the order of $10^{6}$ and $5 \times 10^{2}$ respectively for Venus and Jupiter.

It must be considered, however, that lightning flashes on Earth do not occur in isolation; it has been estimated that about one hundred flashes per second take place over the world as a whole. Since each disturbance lasts an appreciable fraction of a second, the fields are supərimposed, and the total field at a distance may greatly exceed that from a single flash. It is reasonable to assume that a similar state of affairs would exist on the other planets, and this may be a partial explanation of the amplitude discrepancy, particularly for Jupiter, where the storm areas might be much larger than those on Earth. However, it seems necessary also to assume that the scale of the disturbances greatly exceeds that of terrestrial discharges, or that they are of different form, with much more high-frequency energy, or that some form of focusing takes place.

If the noise originates in a multiplicity of storms, its character is difficult to understand. To an observer in outer space, terrestrial noise would be continuous, owing to the integrated effects of a large number of storms. The noise from the planets is reported to consist of bursts, with durations similar to those of typical atmospherics from single lightning flashes. Before the analogy with terrestrial discharges can be accepted, an explanation for the occurrence of discrete bursts must be found. Perhaps these bursts are introduced by fluctuations in the condition of the propagating medium rather than in the activity of the source.

The atmospheric noise observations on which these remarks are based are part of the programme of the Radio Research Board, and this communication is published by permission of the Director of Radio Rosearch of the Department of Scientific and Industrial Research.

Department of Scientific and Industrial Research,

Radio Research Station, Ditton Park, Slough, Bucks. Aug. 14.

${ }^{1}$ Burke, B. F., and Franklin, K. L., J. Geophys. Res., 60, 213 (1955). 'Shain, C. A., Aust. J, Phys., 9, 61 (1956).

${ }^{3}$ Kraus, J. D., Nature, 178, 33 (1956). 